\title{
The microbiota of the vulva and vagina: Ways of washing to optimise the protective function of the vulvo-vaginal microbiota during pregnancy
}

Louise Banga ${ }^{A, B}$ MHIth, BMid, BASOC

${ }^{\mathrm{A}}$ Corresponding Author: louise. banga@gmail.com

${ }^{\mathrm{B}}$ Te Herenga WakaVictoria University of Wellington, Aotearoa New Zealand

\begin{abstract}
Background: The microbiota of the vulva and vagina has a crucial protective function, which is important for all women and has particular significance in pregnancy. Yet this microbiota is part of a delicately balanced ecosystem, susceptible to extrinsic factors which include the simple matter of how women wash themselves. Clinical observation and anecdotal evidence indicate that women in Aotearoa New Zealand have washing practices that may compromise the naturally acidic vulvovaginal environment crucial to optimising the protective function of the microbiota.
\end{abstract}

Aims: The aims of this review are: to determine if there is dissonance between how women are washing their vulva and vagina and recommended washing practices; and to raise awareness of the emerging significance of the vulvo-vaginal microbiota to women's health, particularly in pregnancy.

Method: A literature review was undertaken to discover what is reported (in the published literature) about the ways women wash themselves, products used, and their effect on the vulvo-vaginal microbiota. The evidence behind the "wash with water" recommendation was investigated.

Findings: There is a lack of primary research on ways of vaginal washing used by women in Aotearoa New Zealand. Globally, women are routinely using a variety of products that include soap, antibacterial wipes, gels and baby wipes, and invasive vaginal washing practices such as douching, flannel scrubs and internal soap cleansing. All washing products, including gentle soap but excluding lacticacid based gels, alter $\mathrm{pH}$ levels when used on either the vulva or the vagina. Washing practices that alter vaginal $\mathrm{pH}$ levels can cause a microbial shift into a sub-optimal state that compromises the protective function of the vulvo-vaginal microbiota and is more susceptible to bacterial vaginosis and group B streptococcus vaginal colonisation. The frequency and duration within suboptimal states may be predictors of risk.

Conclusion: There is dissonance between the ways women wash their vulva and vagina, and evidencebased advice to just wash with water. The back-to-basics message "just wash with water" promotes a way of washing that optimises the protective function of the vulvo-vaginal microbiota, while also protecting the integrity of vulval skin, and supporting physiological self-cleaning of the vagina.

Keywords: bacterial vaginosis, microbiota, pregnancy, vagina, vulva, washing

\section{INTRODUCTION}

A deeper understanding is emerging of the unique nature of female-related microbiota, its highly evolved relationship with health outcomes for women, neonates and children, and its role in reproduction (Younes et al., 2018). The maintenance of a healthy vulvo-vaginal microbiota has been recognised as important for optimal pregnancy outcomes (Barthow et al., 2016), for neonatal gastro-intestinal colonisation at birth and for subsequent development of the baby's immune system (Dominguez-Bello et al., 2010; Younes et al., 2018). Yet the vulvo-vaginal microbiota is part of a delicately balanced ecosystem, susceptible to many extrinsic factors that include the simple matter of how women wash themselves.

The rapid development of molecular testing techniques in the last 20 years has led to an exponential information explosion, revealing a dazzling diversity of microbes existing in and on all ecological niches of the human body. Bacteria have now been identified in the placenta, uterus and amniotic cavity in women with healthy pregnancy outcomes, challenging the long-held belief that the human fetus develops in a sterile environment (Younes et al., 2018). Human skin has trillions of resident microbes seen as "partly us, partly not", and is now viewed as a dynamic interface with, rather than a barrier to, the external environment (Hamblin, 2020). Increasingly, dermatological advice promotes washing practices that protect rather than eradicate skin microbiota (Gunter, 2019; Hamblin, 2020). Washing the genital area with water, and avoiding the use of scented soaps, shower gels, bubble baths, shampoos and antiseptics, is recommended by reputable health organisations including New Zealand Family Planning (Stewart, 2019) and 
the United Kingdom National Institute for Health \& Care Excellence (British Association for Sexual Health and HIV, 2014). However, clinical observation and anecdotal evidence indicate that women in Aotearoa New Zealand (Aotearoa NZ) are using soap, anti-bacterial wipes and vaginal flannel scrubs as part of their washing routines of the vulva and vagina.

"Feminine hygiene" products available include soap, "gentle" soap, soap with lactic-acid, body washes, foam, pre-moistened wipes, flavoured suppositories, powders and deodorant sprays, with women encouraged by mass media to wax, shave, douche, steam, sheet mask and flavour their vulva and vagina (Chen et al., 2017; Writes, 2020). Washing routines have become enmeshed with social constructs of cleanliness and wellbeing. The multimillion dollar feminine hygiene industry relies on women feeling their natural state requires "cleaning", with the sinister implication that it is otherwise "dirty". In 2015, for example, Canadian women spent Can $\$ 6.8$ million on vaginal wipes, with the use of vaginal washing products increasing amongst the younger generations (Crann et al., 2018). Why women choose certain washing practices is not well researched nor well understood, even by women themselves (Cottrell, 2010). Influences can be deep-seated, and range from family and local cultural practices, to broader societal influences such as religion, patriarchy, colonisation and marketing strategies inherent to capitalism.

The rather confronting question of "How do you wash your vulva and vagina?" is not routinely discussed with pregnant women. Women can be reluctant to raise the intimate and sensitive topic of the health of their vulva and vagina. This sense of whakamā, or shyness, is acknowledged by many cultures as a barrier to open conversation, effective interventions and more healthful practices (Farage \& Bramante, 2006). In Aotearoa NZ, "recent studies confirm that it is Christianity and Victorian values ... that have suppressed talk about sexual and reproductive health, and marginalised Māori understandings of sexuality, relationships and reproduction" (Green et al., 2016, p. 28). Ambiguous colloquialisms used by women when referring to the vulva and vagina, such as "down there", "mea", "cooch" and "vajayjay", reflect this discomfort and can contribute to confusion when clearer identification is required (Moran, 2020; Wiles, 2017).

This lingering taboo exists within the broader context of fragmented provision of healthcare to the women of Aotearoa $\mathrm{NZ}$, and differential health outcomes between men and women, and between women of different ethnicities and socio-economic levels. Recent petitions for an integrated National Women's Health \& Wellbeing Strategy (Shahtahmasebi, 2021) reflect the critical need to more effectively address the health needs of Māori wāhine and Pasifika women, as well as maternal mental health, the health impact of intimate partner violence, and cervical cancer screening technologies. Vulvo-vaginal health has been stigmatised and under-researched (Younes et al., 2018). At a micro-level, the back-to-basics message "just wash with water" is fundamental to prioritising the health of women and their children, by supporting the physiological processes that ensure resilience and equilibrium of the unique ecosystem of the female microbiota.

This discussion paper considers the value of introducing a new conversation into early pregnancy care and the implications for midwifery practice. As background to this discussion, the vulvo-vaginal microbiota is outlined and findings of a literature review that establishes how women wash and the impact on the vulvo-vaginal microbiota are presented. Bacterial vaginosis (BV), characterised by the disruption of the vaginal microbiota, is one of the most common vulvo-vaginal disorders experienced by women of reproductive age. BV in pregnancy is associated with increased risk of obstetric complications. Hence, this discussion paper also offers clinical considerations and practice points for the management of $\mathrm{BV}$ in pregnancy.

\section{The physiology of the vulvo-vaginal microbiome}

The microbiome is defined as a microbial community associated with a particular environmental niche. The term microbiota refers to the microorganisms found in a specific environment, including bacteria, viruses and fungi (Microbiome Expert Working Group, 2017). Within the vulvo-vaginal microbiome there are multiple and diverse environmental niches, with discretely different microbiota residing within the vulva and vagina. This paper uses the terms "optimal" and "suboptimal" to identify microbiota community states, in favour over other commonly used terms such as "healthy/unhealthy", "eubiosis/ dysbiosis" or "balanced/imbalanced". War analogies, commonly used in microbiology, have been avoided where possible.

\section{The vulva}

The vulva has been described as "a transitional zone between the arid desert of external skin surfaces and the tropical rainforest of the vagina" (Berg \& Davis, 2006, p. 43). Yet the vulva has its own complex ecosystem with multiple habitats within the anatomical structures of the mons pubis, labia majora, labia minora, clitoris, hymen and vaginal vestibule. The appearance of the vulva is unique to each woman, with differences in symmetry, shape, size and colour. Gunter (2019) describes the vulva as the "ultimate multitasker", as it provides physical and microbial protection to the female genital tract, as well as having an integral role in sexual pleasure and birth.

The external mons pubis and labia majora protect the more delicate tissues of the internal structures of the vulva and the vaginal vestibule. The skin has a keratinised epithelium that toughens and waterproofs, is relatively dry and produces hair. In contrast, the skin of the internal labia minora and vaginal vestibule is non-keratinised mucosa, hairless and has a thinner stratum corneum. While skin integrity is maintained by higher moisture levels, anti-microbial fatty substances, and sebum from sebaceous glands, the labia minora and vaginal vestibule are more permeable and susceptible to topical agents (Chen et al., 2017).

Vulval microbiota may include staphylococci, micrococci, diphtheroids, lactobacilli, streptococci, gram-negative rods and yeasts (Chen et al., 2017), with no single species common to all women. Due to the proximity of the vulva to the anal, vaginal and urethral orifices, it is continuously exposed to opportunistic pathogens, and relies on a naturally acidic environment ( $\mathrm{pH} 3.8$ 4.2) to inhibit pathogen colonisation (Bruning et al., 2020).

\section{The vagina}

The vagina is positioned beyond the vaginal vestibule and is composed mainly of mucosa-lined smooth muscle. The vagina has a dynamic, yet delicately balanced, ecosystem that is selfregulating, self-cleaning and resilient (Lewis et al., 2017). Extrinsic factors such as sexual activity, birth, contraception, antibiotics and hygiene practices and products, as well as intrinsic factors that include menstruation, hormonal levels and diet (Barrientos-Durán et al., 2020), can affect this environment. Chen et al. (2017) state: "The normal vaginal flora, acidic vaginal $\mathrm{pH}$, and vaginal discharge are all components of the innate defense mechanisms that protect against vulvo-vaginal 
infections" (p. 60). The vaginal microbiota are relatively stable but can still be variable, with natural fluctuations and significant differences between individuals, making consensus challenging as to what actually constitutes an optimal versus suboptimal microbiota (Microbiome Expert Working Group, 2017).

Vaginal microbiota are commonly classified into five or six community state types (CSTs; Jayaram et al., 2020; Ravel et al., 2011; Sabo et al., 2019). The first four are optimal CSTs, found in $73 \%$ of women and characterised by the dominant presence of one of four common lactobacillus spp. (crispatus, iners, gasseri and jensenii). The remaining CSTs, which are suboptimal, are characterised by fewer lactobacilli and dominated by a diversity of anaerobic bacterium, including the prevotella and megasphaera spp., gardnerella vaginalis, sneathia vaginalis and atopobium vaginae (Romero et al., 2014). The suboptimal CSTs are more susceptible to disease (Barrientos-Durán et al., 2020) and are associated with BV (Ness et al., 2002), group B streptococcus (GBS) vaginal colonisation (Yudin \& Money, 2017), and candidiasis (van der Veer et al., 2019). However, numerous studies report the occurrence of suboptimal CSTs in healthy, asymptomatic women (Younes et al., 2018), challenging the assumption that healthy women are always colonised with high numbers of lactobacilli. Significantly, shifts may occur naturally between CSTs, but it is the frequency and duration within suboptimal states that are considered predictors of the risk of pathogenic overgrowth and infection (Ma et al., 2012).

The maintenance of a slightly acidic environment of $\mathrm{pH}<4.5$ is accepted as a hallmark beneficial activity of lactobacilli, achieved through the production of lactic acid (Younes et al., 2018). Lactobacilli also inhibit pathogen colonisation by forming a physical barrier on epithelial surfaces, producing antimicrobial agents and competing for nutrients, while also influencing immune modulation (Stojanović et al., 2012; Younes et al., 2018).

The vaginal microbiota of healthy pregnant women show significantly higher levels of lactobacilli compared to nonpregnant women, with a reduction of bacterial diversity. As pregnancy advances, levels of lactobacilli increase, with the exception of L. crispatus (Romero et al., 2014). Lactobacilli actively discourage colonisation by pathogenic bacteria that are implicated in adverse pregnancy and neonatal outcomes (Barthow et al., 2016; Marziali et al., 2019), including chlamydia trachomatis, escherichia coli, neisseria gonorrhoea, GBS, and BV-related bacteria such as gardnerella vaginalis.

The vaginal mucosa sheds surface cells approximately every four hours. This frequent shedding is useful for rapid repair, is a nutrition source for resident bacteria, and acts as a decoy to pathogens that attach and are then flushed out as part of the normal vaginal discharge. Involuntary actions of the smooth muscle assist in moving transudate, mucus and sloughed epithelial cells to the vaginal opening (Gunter, 2019). Vaginal discharge commonly measures between one and four millilitres per day, and increases in pregnancy in response to a greater circulating blood volume. This is a highly effective and natural cleansing process.

It is clear that supporting the vulvo-vaginal ecosystem to sustain optimal lactobacilli levels and maintain a naturally acidic environment is crucial to optimising the protective function of the vulvo-vaginal microbiota.

\section{LITERATURE REVIEW}

The objective of the review was to establish, from what has been published, the ways women wash their vulva and vagina, the products commonly used and the impact of these on the vulvovaginal microbiota. The review focussed on regular washing practices and excluded washing practices in the context of menstruation, aesthetics or sexual activity, including non-consensual.

Scholarly sources searched were PubMed, CINAHL, Cochrane Library, Google Scholar, Web of Science, Medline, and Biosis Previews. Search terms used were (vulv* OR vagina* OR genital) AND (washing OR hygien*). Then separate searches were made with the following added: (AND microbio*), (AND New Zealand), (AND (education OR promotion)), (AND 'bacterial vaginosis'). Another search trail was soap AND (washing OR hygiene*) AND (vulv* OR vagina* OR genital) - then AND pH, AND microbio*. A total of 98 papers were identified, with 56 having relevance to the topic. Other material included relevant web resources and recent book publications.

\section{How do women wash their vulva and vagina?}

Globally, women would appear to use a wide variety of practices and products to wash their vulva and vagina. Anti-bacterial wipes, washes/gels, douches and baby wipes were identified as regularly used products in a large, Canadian, cross-sectional survey (Crann et al., 2018). Other common practices found were the use of wet wipes (Farage \& Bramante, 2006), the insertion of a washcloth or tissue to wash the vagina and internal cleansing with soap and water (Esber et al., 2016; Ness et al., 2002). A relatively new, anti-microbial gel wash containing lactic acid is widely available (Bruning et al., 2020) but levels of usage have not yet been studied.

The practice and prevalence of douching, which is the insertion of a device into the vagina to flush liquid, have been comprehensively researched. Commercial preparations can consist of antiseptics, antibacterial preparations, alcohol, surfactant solutions and anti-microbials, while home-made preparations may use herbs, vinegar and water, household bleach, baking soda, yoghurt and water. A large, systematic review revealed that "vaginal douching is a common practice for almost one-third of women in the United States" (Cottrell, 2010, p.102). Other studies show that douching is also routinely practised in Canada (Crann et al., 2018), the United Kingdom (Farage \& Bramante, 2006), Indonesia, Mozambique, South Africa and Thailand (Hilber et al., 2010), Turkey (Hacialioğlu et al., 2009), Brazil (Marconi et al., 2020), Korea (Ahn, 2013), Lebanon (Attieh et al., 2016) and the Netherlands (van der Veer et al., 2019).

No studies were found that specifically address the ways of washing used by women of Aotearoa NZ. Observations from a large, cross-sectional survey of Canadian women (Crann et al., 2018) are arguably the most transferable to the Aotearoa NZ setting. Given the multicultural nature of Aotearoa NZ society, it can be assumed with caution that the literature review findings may be similar and confirm my clinical observations and other anecdotal evidence of similar washing practices by women of Aotearoa NZ.

\section{Impact on the vulvo-vaginal microbiota}

There is an acknowledged paucity of research on how vulval washing practices affect the vulval and vaginal microbiota (Barrientos-Durán et al., 2020; Gunter, 2019), with more attention given to how they affect pH levels. Chen et al. (2017) describe how harsh soaps irritate the vulval skin and mucous membranes and impact the local microbiota. They then detail how exogenous factors, for example the use of soap, detergents, perfumed products, lubricants and spermicides, occlusion with tight clothing or sanitary pads, and depilation practices, can increase $\mathrm{pH}$ levels of the vulval skin. The effect of soap on 
human skin is well researched, with soap alkalinity associated with long-standing alterations to $\mathrm{pH}$ levels and disruption of the resident microbiota (Bruning et al., 2020; Skotnicki, 2018). All soaps, including gentle soaps, strip away natural oils and bacteria that are an important part of the skin's defence (Gunter, 2019). Alterations to skin microbiota can be long-standing after a disruptive event, as observed in a study by Nielsen and Jiang (2019) who found alterations to leg microbiota after swimming in the ocean persisted for at least 24 hours.

Products closer to the $\mathrm{pH}$ of vulval skin, such as lactic acid-based washing gels, appear to have minimal impact (Bruning et al., 2020), and can be recommended when washing is important to prevent inflammation or injury, such as with moderate to severe urinary or fecal incontinence. However, they are not necessary for routine washing of the vulva (Gunter, 2019).

There are numerous studies on the impact of vaginal washing practices on the vaginal microbiota. Douching is consistently associated with shifts to a suboptimal, lactobacillus-reduced microbiota with increased diversity of anaerobic microorganisms (Chen et al., 2017; Cottrell, 2010; Crann et al., 2018; Ness et al., 2002; van der Veer et al., 2019) that promote the growth of C. albicans (van der Veer et al., 2019) and predispose women to BV (Ness et al., 2002). While there is some debate as to the causal direction (Barrientos-Durán et al., 2020; Sabo et al., 2019), the weight of evidence concludes that vaginal douching should be avoided.

The use of gel sanitisers has been associated with higher rates of candidiasis and BV; feminine/baby wipes with higher rates of urinary tract infections; and vaginal moisturiser/lubricant with higher rates of both candidiasis and urinary tract infections (Crann et al., 2018). Bubble bath preparations and antiseptic solutions on the vulva or in the vagina are associated with an increased risk of BV (Chen et al., 2017). Washing inside the vagina (using cloth, lemon juice, soap or detergent) on a regular basis is associated with BV (Sabo et al., 2019), and is also an independent risk factor for vaginal GBS colonisation, a leading cause of early-onset neonatal sepsis (Cools et al., 2016).

\section{Bacterial vaginosis during pregnancy}

Disturbing the vulvo-vaginal microbiota has particular significance during pregnancy. BV is the most common cause of abnormal vaginal discharge in reproductive women (Lewis et al., 2017; Ma et al., 2012), with an estimated 25\% of pregnant women in the US affected (Stojanović et al., 2012). There is currently no published Aotearoa NZ data, with routine reporting of $\mathrm{BV}$ not required. BV is generally characterised by disruption of the vaginal microbiota equilibrium, with significantly reduced numbers of lactobacilli, decreased lactic acid concentrations, and elevated $\mathrm{pH}$ levels (Petrova et al., 2015). This supports an overgrowth of pathogenic bacteria such as gardnerella vaginalis, mycoplasma hominis, and/or mobiluncus species (Lamont, 2015). Ma et al. (2012, p. 2) speculate that "the disturbed state itself may constitute the clinical syndrome known as BV, which as a disruption of ecologica equilibria is believed to increase the risk of invasion by infectious agents". Despite decades of research the specific aetiology of BV remains controversial (Muzny \& Kardas, 2020).

BV in early pregnancy has been classified as a risk factor for preterm birth (Integrative Human Microbiome Research Network Consortium, 2019; Lamont, 2015; Leitich et al., 2003), although the precise causal pathway is unclear. BV is also associated with an increased risk of premature rupture of fetal membranes and chorioamnionitis (Jayaram et al.,
2020). Furthermore, BV facilitates the acquisition of sexually transmitted infections such as gonorrhoea, chlamydia and HIV (Petrova et al., 2015). There is some speculation that the thick, multispecies biofilm typical of BV may play a part in bacterial transmission from vagina to fetal membranes (Jayaram et al., 2020; Lim et al., 2010). Until a more precise aetiology of BV is known, management in pregnancy remains an important issue (Livengood, 2009; Shimaoka et al., 2019).

\section{Clinical considerations}

The classic presentation of BV is of a moderately increased vaginal discharge that is thin, whitish-grey, sometimes "frothy", and adherent to the vaginal wall (Table 1). There is usually a distinctive "fishy" odour. Symptoms often self-resolve; however, it is recommended that symptomatic BV is treated in pregnancy (Perkins, 2019; Yudin \& Money, 2017). Anti-microbial treatment aims "to improve symptoms and possibly reduce the complications associated with BV in pregnancy" (Jayaram et al., 2020, p.4). Treatment can be commenced immediately with pregnant women who are clearly symptomatic, or once diagnosis is confirmed (Perkins, 2019; Yudin \& Money, 2017).

The routine screening for $\mathrm{BV}$ and treatment of asymptomatic women with an incidental BV diagnosis are not currently supported in pregnancy (Perkins, 2019; US Preventive Services Task Force [USPSTF], 2020). Some practitioners may choose to screen pregnant women with a history of previous preterm birth $<35$ weeks and treat accordingly (Jayaram et al., 2020; Yudin \& Money, 2017). However, the evidence is inconclusive on the benefits of treating asymptomatic women who test positive within this group (Lamont, 2015; USPSTF, 2020).

Diagnosis of BV from a Gram stain-based test (the current gold standard) is determined by the relative concentration of lactobacilli (long gram-positive rods) to gram-negative and gram-variable rods and cocci, using the Nugent score. This method of diagnosis has led to speculation that there may also be over-diagnosis (Ma et al., 2012), with healthy, asymptomatic women who have innate low levels of lactobacillus potentially receiving a $\mathrm{BV}$ diagnosis. New molecular tests for diagnosis represent promising point-of-care diagnosis (American College of Obstetricians and Gynecologists [ACOG], 2020).

Recurring BV is common, with up to $30 \%$ of women experiencing a recurrence within three months and 50\% within twelve months of treatment (ACOG, 2020). There are few data available regarding optimal management strategies for women with persistent or recurrent BV. Retreatment is an acceptable initial approach (ACOG, 2020; Perkins, 2019), before considering medical referral. Switching between approved antibiotic medications and modes of administration may then be considered (Remaly, 2020). New treatments being explored emphasise biofilm disruption; the re-establishment of normal acidic vaginal $\mathrm{pH}$, for example with microbiota transplants and lactic acid gel (Remaly, 2020); and treatments that manipulate and restore an optimal vulvo-vaginal microbiota, for example probiotic therapies (Barthow et al., 2016).

$\mathrm{BV}$ is a perplexing condition but, due to associations with increased risk of obstetric complications, should not be underestimated. Establishing clear prevention strategies without precise aetiology is difficult, but consideration of washing practices would appear to be reasonable. Avoiding washing practices associated with an increased risk of $\mathrm{BV}$, and washing in ways that maintain the naturally acidic vulvo-vaginal $\mathrm{pH}$, may reduce the frequency of shifts from an optimal to suboptimal vaginal environment and minimise opportunities for BV-related 
bacteria to establish. Targeting women's modifiable behaviour in pregnancy to reduce the chance of $\mathrm{BV}$ and the appropriate management of $\mathrm{BV}$ are both relevant to midwifery practice.

\section{Table 1. Practice points regarding bacterial vaginosis*}

\section{Identifying}

Woman concerned about vaginal discharge symptoms:

- Increased vaginal discharge

- Discharge is whitish grey and may be "frothy" - Distinctive "fishy" odour

\section{Assessment}

Take a history and identify:

- Volume

- Colour

- Frequency (constant or intermittent)

- Odour

- Pain or itchiness

- Pelvic or urinary symptoms

- Any contact with STI

- General history

Routine screening is not currently supported.

Tests

- Examination of vulva and vagina (speculum)

- Take a high vaginal swab for Gram stain and culture

- Take a vulvo-vaginal swab to test for chlamydia, gonorrhoea and trichomonas

- Check antenatal blood tests for serology results

BV is not considered an STI but other STIs need to be excluded.

Treatment

- If symptomatic of BV, treatment of pregnan women in Aotearoa NZ can be offered and commenced with informed consent before swab results are back.

- Metronidazole $400 \mathrm{mg}$ bd for 7 days. Advise to take with meals to reduce possible side effects such as nausea or upset stomach.

- Metronidazole $2 \mathrm{gm}$ stat (5 x 400mg) remains a valid option if there are issues of compliance or at woman's request, but is less efficacious with a higher rate of relapse.

- Advise women to contact either midwife or GP if symptoms persist or return.

Please note that treatment of BV with pharmacological boric acid or metronidazole vaginal suppositories is not recommended for use in pregnancy.

\section{A new conversation in pregnancy?}

It appears that there is value in the integration of a conversation about vulvo-vaginal washing practices into early pregnancy care. It will be important that this highly intimate conversation is approached with sensitivity, and in a way that honours the diversity of meaning and experiences for all women of Aotearoa NZ. Midwives are well placed to support women through this conversation, having a practice model that enables women to control the determinants of their health, and that focusses on building a trusting relationship.

There are various ways to integrate this conversation into antenatal care and discuss ways of washing that optimise the protective function of the vulvo-vaginal microbiota during pregnancy. Taking the time to discover and then use the words women are comfortable with to identify their genital anatomy, while at the same time taking the opportunity to demystify and inform, may encourage women to talk more freely about their bodies in the future.
The "just wash with water" message sits easily alongside "always wipe from the front to the back after having a bowel motion", and "pass urine soon after sexual activity" self-care advice. These messages are usually delivered in the context of pregnant women being more susceptible to urinary tract infections (Betschart et al., 2020). As health practitioners discussing healthy ways of washing, being aware of the deep-seated nature and diversity of factors that influence a woman's attitude to her genital health is important. Women may have changed their usual practices in response to being pregnant; for example, not using condoms, washing more or washing less, using different products, changing sexual behaviours and frequency etc. There may be a history of sexual or genital abuse, or fear-based behaviours that include blocking out or, conversely, acutely focussing on genital health. With current levels of poverty and homelessness in Aotearoa NZ, there may also be issues with easy access to washing water.

The "just wash with water" message has synchronicity with advances in skin care that prioritise the preservation of microbiota, and with environmental and anti-consumerist movements, while challenging the ethics of capitalism and the advertising industry. These are issues that may also trigger women to reconsider how they care for their vulva and vagina.

Figure 1. Author's visual tool to help introduce the topic of vulvo-vaginal care

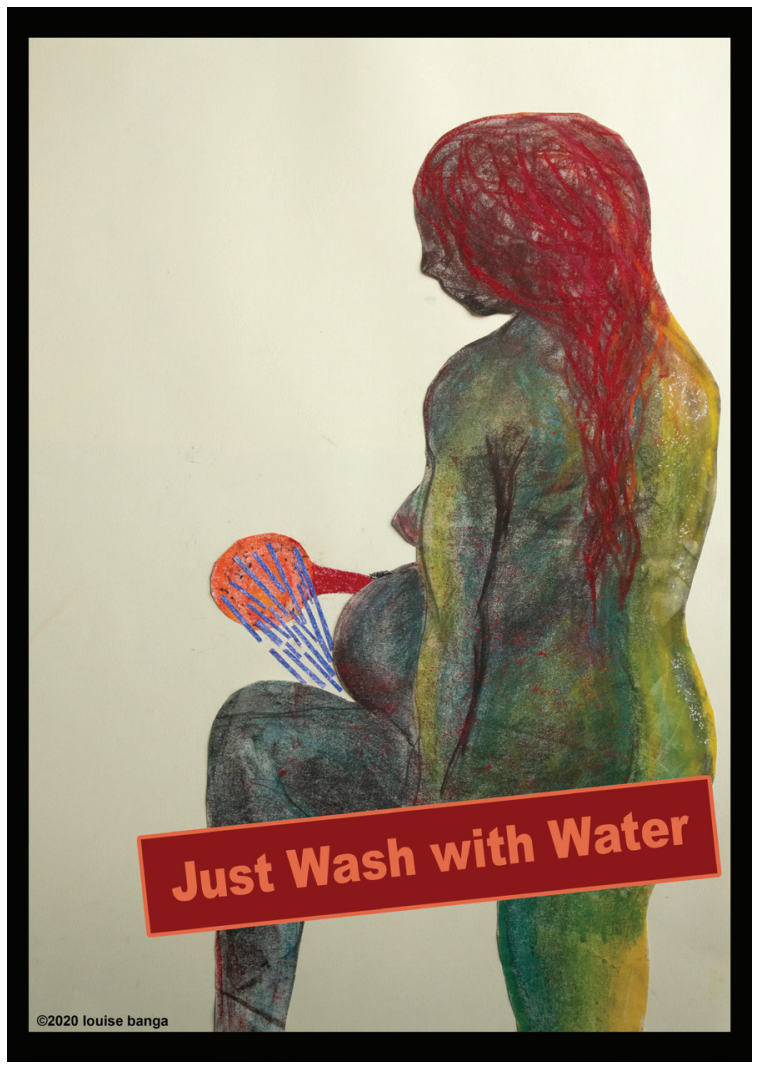

The graphic (Figure 1) was created by the author, and permission is given for it to be used as a visual tool to help introduce the topic, or display as a poster in antenatal clinics. There are also some excellent YouTube clips with evidence-based scripts that discuss vulvo-vaginal care; two examples are Pelvic love: vulval/ vaginal hygiene - how to keep the vulva happy (https://www. youtube.com/watch? $v=$ BzUvimxoWWc) from the United Kingdom and Madge the vag (https://www.youtube.com/ watch?v=f35fxT0sHEs) from the United States. No Aotearoa 
NZ-based resources have been found on this subject, so development of context-specific educational resources for women and midwives (including multilingual versions) would be helpful. Integrating the "just wash with water" message into other Ministry of Health pregnancy resources may be a useful first step.

There is growing scientific interest in female-related microbes and their relationship to health outcomes for pregnant women. However, more research is needed, looking specifically at the Aotearoa NZ context, to explore the factors that influence women's choices when considering washing practices of the vulva and vagina.

\section{CONCLUSION}

Women are regularly using a variety of products and ways of washing that are not evidence-based and do not follow professional recommendations for optimal vulvo-vaginal health. All washing products, including gentle soap but excluding lactic acid-based gels, alter protective $\mathrm{pH}$ levels when used on either the vulva or the vagina. Washing practices that alter vaginal $\mathrm{pH}$ levels can cause a microbial shift into a suboptimal state that is more susceptible to pathogenic overgrowth and infection. High frequency and longer duration within suboptimal states are thought to be predictors of risk. Douching and invasive vaginal washing practices increase the risk of BV and GBS vaginal colonisation, conditions associated with an increased risk of obstetric and neonatal complications. Gentle washing of the vulva with water and avoidance of any vaginal washing are best practice to help maintain optimal vulvo-vaginal microbiota and support the protective function. Vulval washing with water also protects the integrity of vulval skin, and supports physiological self-cleaning of the vagina. This paper argues that discussion on women's ways of intimate washing is an important aspect of pregnancy care and the simple "just wash with water" message is evidence-based and requires more resources to support its use.

\section{CONFLICT OF INTEREST DISCLOSURE}

The author declares that there are no conflicts of interest.

\section{Key points}

- The microbiota of the vulva and vagina has a crucial protective function, yet is part of a delicately balanced ecosystem susceptible to extrinsic factors that include the simple matter of how women wash themselves.

- $\quad$ Evidence supports that gentle washing of the vulva with water and avoidance of any vaginal washing products are best practice to maintain an optimal vulvo-vaginal microbiota.

- The "just wash with water" message sits easily alongside advice such as "always wipe from the front to the back after having a bowel motion" and "pass urine soon after sexual activity", delivered in the context of pregnant women being more susceptible to urinary tract infections.

\section{REFERENCES}

Ahn, S. (2013). Feminine hygiene and vaginal douche practices in middle-aged women. Korean Journal of Women Health Nursing, 19(4), 285-294. https://doi.org/10.4069/kjwhn.2013.19.4.285

American College of Obstetricians and Gynecologists. (2020). Bacterial vaginitis. MicroRounds Series. https://www.acog.org/education-andevents/emodules/bacterial-vaginosis-microrounds

Attieh, E., Maalouf, S., Roumieh, D., Abdayem, P., AbiTayeh, G., \& Kesrouani, A. (2016). Feminine hygiene practices among female patients and nurses in Lebanon. Reproductive Health, 13, 59. https://doi. org/10.1186/s12978-016-0182-4

Barrientos-Durán, A., Fuentes-López, A., de Salazar, A., Plaza-Díaz, J., \& García, F. (2020). Reviewing the composition of vaginal microbiota: Inclusion of nutrition and probiotic factors in the maintenance of eubiosis. Nutrients, 12(2), 419. https://doi.org/10.3390/nu12020419

Barthow, C., Wickens, K., Stanley, T., Mitchell, E., Maude, R., Abels, P., Purdie, G., Murphy, R., Stone, P., Kang, J., Hood, F., Rowden, J., Barnes, P., Fitzharris, P., Craig, J., Slykerman, R., \& Crane, J. (2016). The Probiotics in Pregnancy Study (PiP Study): Rationale and design of a double-blind randomised controlled trial to improve maternal health during pregnancy and prevent infant eczema and allergy. BMC Pregnancy and Childbirth, 16, 133. https://doi.org/10.1186/s12884-016-0923-y

Berg, R., \& Davis, C. (2006). Microbial ecology of the vulva. In M. Farage \& H. Maibach (Eds.), The vulva: Anatomy, physiology and pathology (1st ed., pp. 43-55). Informa Healthcare.

Betschart, C., Albrich, W., Brandner, S., Faltin, D., Kuhn, A., Surbek, D., \& Geissbuehler, V. (2020). Guideline of the Swiss Society of Gynaecology and Obstetrics (SSGO) on acute and recurrent urinary tract infections in women, including pregnancy. Swiss Medical Weekly, 150, w20236. https://doi.org/10.4414/smw.2020.20236

British Association for Sexual Health and HIV. (2014). UK national guideline on the management of vulval conditions. National Institute for Health and Care Excellence. https://www.evidence.nhs.uk/ search?q=vulval+

Bruning, E., Chen, Y., McCue, K., Rubino, J., Wilkinson, J., \& Brown, A. (2020). A 28 day clinical assessment of lactic acid-containing antimicrobial intimate gel wash formulation on skin tolerance and impact on the vulvar microbiome. Antibiotics, 9(2), 55. https://doi. org/10.3390/antibiotics 9020055

Chen, Y., Bruning, E., Rubino, J., \& Eder, S. (2017). Role of female intimate hygiene in vulvovaginal health: Global hygiene practices and product usage. Women's Health, 13(3), 58-67. https://doi. org/10.1177/1745505717731011

Cools, P., Jespers, V., Hardy, L., Crucitti, T., Delany-Moretlwe, S., Mwaura, M., Ndayisaba, G., van de Wijgert, J., \& Vaneechoutte, M. (2016). A multi-country cross-sectional study of vaginal carriage of Group B Streptococci (GBS) and Escherichia coli in resource-poor settings: Prevalences and risk factors. PloS one, 11(1), e0148052. https:// doi.org/10.1371/journal.pone.0148052

Cottrell, B. (2010). An updated review of evidence to discourage douching. The American Journal of Maternal/Child Nursing, 35(2), 102107. https://doi.org/10.1097/NMC.0b013e3181cae9da

Crann, S., Cunningham, S., Albert, A., Money, D., \& O’Doherty, K. (2018). Vaginal health and hygiene practices and product use in Canada: A national cross-sectional survey. BMC Women's Health, 18, 52. https:// doi.org/10.1186/s12905-018-0543-y

Dominguez-Bello, M., Costello, E., Contreras, M., Magris, M., Hidalgo, G., Fierer, N., \& Knight, R. (2010). Delivery mode shapes the acquisition and structure of the initial microbiota across multiple body habitats in newborns. Proceedings of the National Academy of Sciences of the United States of America, 107(26), 11971-11975. https://doi. org/10.1073/pnas.1002601107

Esber, A., Turner, A., Mopiwa, G., \& Norris, A. (2016). Intravaginal practices among a cohort of rural Malawian women. Sexual Health 13(3), 275-280. https://doi.org/10.1071/SH15139

Farage, M., \& Bramante, M., (2006). Genital hygiene: culture, practices, and health impact. In M. Farage \& H. Maibach (Eds.), The vulva: Anatomy, physiology and pathology (1st ed., pp. 183-216). Informa Healthcare.

Green, J., Tipene, J., \& Davis, K. (2016). Mana tangata whenua: National guidelines for sexual and reproductive health promotion with Mãori (1st ed).Te Whāriki Takapou. https://tewhariki.org.nz/assets/ National_Guidelines_for_SRH_Promotion_with_Māori.pdf

Gunter, J. (2019). The vagina bible: The vulva and the vagina - Separating the myth from the medicine. Piatkus. 
Hacialioğlu, N., Nazik, E., \& Kiliç, M. (2009). A descriptive study of douching practices in Turkish women. International Journal of Nursing Practice, 15(2), 57-64. https://doi.org/10.1111/j.1440-172X.2009.01735.x Hamblin, J. (2020). Clean. The new science of skin and the beauty of doing less. Penguin Random House.

Hilber, A., Hull, T., Preston-Whyte, E., Bagnol, B., Smit, J., Wacharasin, N., \& Widyantoro, N. (2010). A cross cultural study of vaginal practices and sexuality: Implications for sexual health. Social Science \& Medicine, 70(3), 392-400. https://doi.org/10.1016/j.socscimed.2009.10.023

Integrative Human Microbiome Research Network Consortium. (2019). The integrative human microbiome project. Nature 569, 641-648. https://doi.org/10.1038/s41586-019-1238-8

Jayaram, P., Mohan, M., \& Konje, J. (2020). Bacterial vaginosis in pregnancy - a storm in the cup of tea. European Journal of Obstetrics \& Gynecology and Reproductive Biology, 253, 220-224. https://doi. org/10.1016/j.ejogrb.2020.08.009

Lamont R. F. (2015). Advances in the prevention of infection-related preterm birth. Frontiers in Immunology, 6, 566. https://doi.org/10.3389/ fimmu.2015.00566

Leitich H., Bodner-Adler B., Brunbauer M., Kaider A., Egarter C., \& Husslein P. (2003). Bacterial vaginosis as a risk factor for preterm delivery: A meta-analysis. American Journal of Obstetrics \& Gynecology, 189(1), 139-147. https://doi.org/10.1067/mob.2003.339 Lewis, F., Bogliatto, F., \& van Beurden, M. (2017). A practical guide to vulval disease: Diagnosis and management. Wiley Blackwell.

Lim, K., Brooks, H., McDougal, R., Burton, J., Devenish, C., \& De Silva, T. (2010). Is there a correlation between bacterial vaginosis and preterm labour in women in the Otago region of New Zealand? Australian and New Zealand Journal of Obstetrics and Gynaecology, 50, 226-229. https://doi.org/10.1111/j.1479-828X.2010.01149.x

Livengood, C. (2009). Bacterial vaginosis: an overview for 2009. Reviews in Obstetrics \& Gynecology, 2(1), 28-37. PMC2672999

Ma, B., Forney, L. J., \& Ravel, J. (2012). Vaginal microbiome:

Rethinking health and disease. Annual review of microbiology, 66, 371-

389. https://doi.org/10.1146/annurev-micro-092611-150157

Marconi, C., El-Zein, M., Ravel, J., Ma, B., Lima, M., Carvalho, N.,

Alves, R., Parada, C., Leite, S., Giraldo, P., Gonçalves, A., Franco, E., \& Silva, M. (2020). Characterization of the vaginal microbiome in women of reproductive age from 5 regions in Brazil. Sexually Transmitted Diseases, 47(8), 562-569. https://doi.org/10.1097/OLQ.0000000000001204

Marziali, G., Foschi, C., Parolin, C., Vitali, B., \& Marangoni, A. (2019). In-vitro effect of vaginal lactobacilli against group B Streptococcus. Microbial Pathogenesis, 136, 103692. https://doi.org/10.1016/j. micpath.2019.103692

Microbiome Expert Working Group. (2017). Unlocking the microbiome. Microbiology Society. https://microbiologysociety.org/our-work/themicrobiome/unlocking-the-microbiome-report.html

Moran, C. (2020). More than a woman. Penguin Random House.

Muzny, C., \& Kardas, P. (2020). A narrative review of current challenges in the diagnosis and management of bacterial vaginosis. Sexually Transmitted Diseases, 47(7), 441-446. https://doi.org/10.1097/ OLQ.0000000000001178

Ness, R., Hillier, S., Richter, H., Soper, D., Stamm, C., McGregor, J., Bass, D., Sweet, R., \& Rice, P. (2002). Douching in relation to bacterial vaginosis, lactobacilli, and facultative bacteria in the vagina. Obstetrics \& Gynecology, 100(4), 765-772. https://doi.org/10.1016/s00297844(02)02184-1

New Zealand Sexual Health Service. (2017). Vaginal discharge: Management summary. https://www.nzshs.org/docman/guidelines/ management-of-sexual-health-conditions/vaginal-discharge/182-vaginaldischarge-summary/file

Nielsen, M., \& Jiang, S. (2019). Alterations of the human skin microbiome after ocean water exposure. Marine Pollution Bulletin, 145, 595-603. https://doi.org/10.1016/j.marpolbul.2019.06.047

Perkins, N. (2019, January 25). Vaginal discharge and bacterial vaginosis [audio podcast episode]. Goodfellow Unit. https://www.goodfellowunit. org/podcast/vaginal-discharge-and-bacterial-vaginosis

Petrova, M., Lievens, E., Malik, S., Imholz, N., \& Lebeer, S. (2015).

Lactobacillus species as biomarkers and agents that can promote various aspects of vaginal health. Frontier Physiology, 6, 81. https://doi. org/10.3389/fphys.2015.00081
Ravel, J., Gajer, P., Abdo, Z., Schneider, M., Koenig, S., McCulle, S., Karlebach, S., Gorle, R., Russell, J., Tacket, C., Brotman, R., Davis, C., Ault, K., Peralta, L., \& Forney, L. (2011). Vaginal microbiome of reproductive-age women. Proceedings of the National Academy of Sciences, 108(Suppl. 1), 4680-4687. https://doi.org/10.1073/pnas. 1002611107

Remaly, J. (2020, September 28). Consider switching up treatment regimens for recurrent bacterial vaginosis. Virtual conference coverage. International Society for the Study of Vulvo-vaginal Disease. https:// www.mdedge.com/obgyn/article/229144/gynecology/consider-switchingtreatment-regimens-recurrent-bacterial-vaginosis

Romero, R., Hassan, S., Gajer, P., Tarca, A., Fadrosh, D., Nikita, L., Galuppi, M., Lamont, R., Chaemsaithong, P., Miranda, J., Chaiworapongsa, T., \& Ravel, J. (2014). The composition and stability of the vaginal microbiota of normal pregnant women is different from that of non-pregnant women. Microbiome, 2, 4. https://doi. org/10.1186/2049-2618-2-4

Sabo, M., Balkus, J., Richardson, B., Srinivasan, S., Kimani, J., Anzala, O., Schwebke, J., Feidler, T., Fredricks, D., \& McClelland, R. (2019). Association between vaginal washing and vaginal bacterial concentrations. PLoS One, 14(1), e0210825. https://doi.org/10.1371/journal. pone.0210825

Shahtahmasebi, Z. (2021, March 17). Petition launched for a $\$ 6$ million women's health strategy. New Zealand Doctor. https://www.nzdoctor.co.nz/ article/undoctored/petition-launched-6-million-womens-health-strategy

Shimaoka, M., Yo, Y., Doh, K., Kotani, Y., Suzuki, A., Tsuji, I., Mandai, M., \& Matsumura, N. (2019). Association between preterm delivery and bacterial vaginosis with or without treatment. Scientific reports, 9, 509. https://doi.org/10.1038/s41598-018-36964-2

Skotnicki, S. (2018). Beyond Soap: The real truth about what you are doing to your skin and how to fix it for a beautiful, healthy glow. Penguin Random House.

Stewart, R. (2019, December 16). Five things people need to stop putting in their vaginas in 2020. Newshub. https://www.newshub.co.nz/home/ lifestyle/2019/12/five-things-people-need-to-stop-putting-in-theirvaginas-in-2020.html

Stojanović, N., Plećaš, D., \& Plešinac, S. (2012). Normal vaginal flora, disorders and application of probiotics in pregnancy. Archives of Gynecology \& Obstetrics, 286, 325-332. https://doi.org/10.1007/s00404012-2293-7

US Preventive Services Task Force. (2020). Screening for bacterial vaginosis in pregnant persons to prevent preterm delivery: US Preventive Services Task Force recommendation statement. Journal of American Medical Association, 323(13), 1286-1292. https://doi.org/10.1001/ jama.2020.2684

van der Veer, C., Bruisten, S., van Houdt, R., Matser, A., Tachedjian, G., van de Wijgert, J., de Vries, H., \& van der Helm, J. (2019). Effects of an over-the-counter lactic-acid containing intra-vaginal douching product on the vaginal microbiota. BMC Microbiology, 19, 168. https://doi. org/10.1186/s12866-019-1545-0

Wiles, S. (2017). Antibiotic resistance: The end of modern medicine? Bridget Williams Books.

Writes, E. (2020, October 16). Don't put vanilla melts in your vagina. https://emilywrites.substack.com/p/dont-put-vanilla-melts-in-your-vagina Younes, J., Lievens, E., Hummelen, R., van der Westen, R., Reid, G., \& Petrova, M. (2018). Women and their microbes: The unexpected friendship. Trends in Microbiology, 26(1), 16-32. https://doi. org/10.1016/j.tim.2017.07.008

Yudin, M., \& Money, D. (2017). Practice Guideline: No. 211 Screening and management of bacterial vaginosis in pregnancy. Journal of Obstetrics and Gynaecology Canada, 39(8), e184-e191. https://doi. org/10.1016/j.jogc.2017.04.018

\section{Accepted for Publication June 2021}

Banga, L. (2021). The microbiota of the vulva and vagina: Ways of washing to optimise the protective function of the vulvo-vaginal microbiota during pregnancy. New Zealand College of Midwives Journal, 57, 34-40.

https://doi.org/10.12784/nzcomjnl57.2021.5.34-40 